\title{
Vision périphérique des objets : performances et niveau de conscience. Une application à la dégénérescence maculaire liée à l'âge
}

Michèle FABRE-THORPE

Centre de Recherche Cerveau et Cognition (UMR 5549)

Faculté de Médecine

133 route de Narbonne

31062 Toulouse

Tel : 0562172807
Sous-thèmes dont relève ce projet :

Vision

Temps

Mémoire

Autres troubles: DMLA, Stargardt, psychopharmacologie (effets du Vigabatrin)

\section{Equipes partenaires}

- Neuropsychologie Expérimentale (Lille 2) - CHU Lille Hôpital Salengro- Exploration fonctionnelle de la vision, 59037 Lille

+ Institut Jean Nicod - 1 bis, Rue Löwendal - 75007 Paris, France

\section{Résumé signalétique}

Ce projet rassemblait des neurophysiologistes, des psychologistes expérimentaux, des cliniciens et des philosophes. Le but était d'évaluer les capacités de la rétine périphérique face à des stimuli biologiquement pertinents comme des scènes naturelles ou des objets isolés, puis de tenter de déterminer comment ces résultats pourraient permettre d'améliorer l'autonomie de patients porteurs de certaines pathologies les rendant totalement tributaires des fonctions résiduelles périphériques (dégénérescence maculaire liée à l'âge ou maladie de Stargardt). Face à des stimuli naturels, peu de choses étaient connues sur les capacités de la rétine périphérique ; il s'est donc agi d'évaluer les performances de sujets sains et de patients dans des tâches visuelles de haut niveau utilisant des stimuli naturels périphériques et de déterminer certaines caractéristiques des mécanismes cérébraux sous-jacents. Parallèlement, l'une des interrogations communes aux chercheurs impliqués dans ce projet concernait l'aspect explicite ou implicite de la reconnaissance d'objets effectuée à partir d'informations visuelles périphériques. Les réponses motrices dans de telles tâches cognitives de haut niveau pouvaient-elles être déclenchées à partir de représentations mentales non conscientes ? À plus long terme, pourrait-on utiliser ces capacités implicites dans la rééducation de pathologies visuelles.?

Les résultats obtenus ont permis de mettre en évidence : (1) un parallélisme massif des traitements rapides du système visuel qui peut analyser, sans coût temporel, 2 images naturelles différentes, (2) les structures mises en jeu dans la catégorisation rapide d'un stimulus excentré (3) une reconnaissance implicite jusqu'à $60^{\circ}$ d'excentricité alors que la reconnaissance explicite est très affectée à cette même excentricité et (4) la détérioration de la reconnaissance implicite à $60^{\circ}$ chez des patients épileptiques traités à la vigabatrine.

Mots-clés : Vision périphérique • catégorisation • identification • représentations nonconscientes * vision résiduelle.

Nombre de participants : 11

Nombre d'homme-mois : 56 


\section{Rappel des enjeux et objectifs fixés à l'origine}

Ce projet avait été conçu à partir de données suggérant que certains processus de traitement visuels rapides et automatiques seraient beaucoup plus sophistiqués que ce qui est généralement admis et pourraient être utilisés en rétine périphérique. Une activité cognitive de haut niveau, telle que la détection d'un animal, pourrait en quelque sorte être réalisée en «blindsight» à partir de la première vague d'informations visuelles sans aucune exploration oculaire du stimulus et avant qu'une réelle "perception» détaillée et consciente de l'objet n'ait encore eu lieu. L'objectif principal du projet était d'évaluer, chez des sujets sains et chez des patients souffrant de handicaps visuels, les capacités de la rétine périphérique face à des stimulus naturels. L'un des objectifs à long terme était de permettre l'utilisation d'un potentiel visuel sous-estimé et non exploité dans la rééducation d'un certain type de patients malvoyants.

Plusieurs questions étaient posées abordant les capacités de la rétine périphérique jusqu'à de très larges excentricités :

1. Quelles sont les performances de sujets normaux et de patients présentant des pathologies visuelles dans des tâches de catégorisation ou de reconnaissance d'objets lorsque la réponse motrice à fournir s'appuie sur un mode de traitement rapide (non-conscient ?) de l'information visuelle?

\section{Résumé des résultats effectivement atteints}

La collaboration toute nouvelle qui s'est établie entre les équipes de Michèle Fabre-Thorpe et de Muriel Boucart a été très fructueuse. De nombreux résultats ont été obtenus sur le fonctionnement de la rétine périphérique face à des stimuli naturels.

Au Cerco à Toulouse, l'étude des capacités de catégorisation de l'homme jusquà des excentricités extrêmes (>80\%) a été publiée. Pour des présentations parafovéales, nous avons montré que ni la latence de l'activité cérébrale différentielle (qui apparaît à $150 \mathrm{~ms}$ entre essais cibles et distracteurs lors des présentations centrales) ni son amplitude ne sont affectées par la latéralisation du stimulus, et nous avons localisé les dipôles responsables. Enfin, nous avons montré l'important parallélisme de traitement qui existe dans le système visuel face à deux scènes naturelles présentées simultanément dans chacun des deux hémichamps. Ces résultats ont été publiés dans Nature Neuroscience et choisis comme objet d'un Highlight dans «Nature Neuroscience Reviews, 2002, 3, 589». Les limites de ce parallélisme sont actuellement recherchées lorsque les 2 scènes sont présentées dans le même hémichamp ou lorsque 4 scènes sont présentées simultanément.

Une salle dédiée à l'étude de la vision périphérique est en cours d'installation à l'Hôpital Purpan (locaux de l'IFR Sciences du Cerveau de Toulouse). La $2^{\mathrm{e}}$ tranche de crédit va nous permettre d'achever sa mise au point.

Dans le service d'Exploration Fonctionnelle de la Vision du CHRU de Lille, une salle d'étude de la vision périphérique a été installée. Les capacités de reconnaissance explicite et implicite ont été testées chez le sujet sain à grande excentricité
2. Quelles sont les caractéristiques des mécanismes neuronaux sous-jacents à la catégorisation rapide de stimuli naturels périphériques? Notamment quelles sont les limites du parallélisme de traitement.

3. Quel est le niveau de conscience des représentations soustendant la perception rapide des objets et des formes en périphérie?

4. Peut-on apprendre à mieux utiliser les capacités résiduelles de la rétine périphérique pour améliorer la vie quotidienne de certains patients (DMLA, stargardt). Quels seraient les bénéfices d'une stratégie spécifique de déploiement de l'attention spatiale.

Dans certaines pathologies affectant la rétine centrale, l'identification de lettres et par extension la lecture qui nécessitent une grande acuité visuelle, sont très perturbées, mais les informations visuelles périphériques pourraient être suffisantes pour assurer de nombreuses tâches quotidiennes et même des tâches complexes de reconnaissance d'objets. L'autonomie de certains patients pourrait ainsi se trouver améliorée si une rééducation adaptée leur permettait d'apprendre à utiliser de nouvelles stratégies pour faire appel à des représentations d'objets non-conscientes.

$\left(60^{\circ}\right)$. La reconnaissance explicite exige des sujets qu'ils récupèrent consciemment un événement spécifique déjà vu tandis que la reconnaissance implicite s'exprime par une facilitation de la performance qui ne nécessite pas la récupération consciente d'un événement antérieur. Une reconnaissance implicite a été observée à 30 et $60^{\circ}$ mais l'amorçage conceptuel n'a été observé que jusqu'à $30^{\circ}$ d'excentricité. L'effet d'amorçage pour les images identiques indique qu'une reconnaissance implicite, non consciente, est possible à $60^{\circ}$ d'excentricité.

Lobtention des autorisations des comités d'éthique a considérablement retardé l'étude des patients DMLA et Stargardt. En attendant, des patients épileptiques présentant des troubles visuels liés à la prise de conscience des informations périphériques (secondaires au traitement par la vigabatrin) ont été étudiés. Les capacités de reconnaissance d'objets (reconnaissance implicite ou de catégorisation rapide d'objets) encore préservées à $30^{\circ}$ d'excentricité (70 \% correct) sont très perturbées en grande périphérie.

Les interactions entre neurophysiologistes, neuropsychologues et cliniciens ont été fructueuses. La réunion prévue avec les philosophes sur le thème «perception périphérique et conscience» va être organisée dans un futur proche. 


\section{Publications issues du projet}

Thorpe S.J., Gegenfurtner K., Fabre-Thorpe M. \& Bülthoff H.H. (2001). Detection of animals in natural images using far peripheral vision. European Journal of Neuroscience, 14, 869-876.

Fize D., Fabre-Thorpe M., Richard G., Doyon B. \& Thorpe S. (soumis). Rapid categorisation of foveal and extrafoveral natural images : Associated ERPs and effect of lateralisation. Experimental Brain Research.

Rousselet G., Fabre-Thorpe M. \& Thorpe S.J. Parallel processing in high level categorization of natural images. Nature Neuroscience, 5 , 629-630.

Boucart M., Fabre-Thorpe M., Thorpe S., Arndt C. \& Hache J.C. (2001). Covert object recognition at large visual eccentricity. Proceedings of the 1st meeting of the Vision Science Society (Sarasota, Florida, USA). Journal of Vision, 1 (3), 471a. http://journalofvision.org/
Boucart M. \& Fabre-Thorpe M., Arndt, C. \& Derambure, P. (2001). Visual impairment in far periphery with Vigabatrin : visual, attentional or recognition deficit? Perception, 30 Suppl, 107a.

\section{Article de vulgarisation}

Boucart M. \& Fabre-Thorpe M., Apprendre à voir du coin de l'œil Science et Vie, Hors série «L'ail et la vision» 216, 153-157 
